\title{
Multiplicités : la série énumérative dans L'Acacia
}

\section{Michel Sandras}

\section{(2) OpenEdition}

Journals

Édition électronique

URL : http://journals.openedition.org/ccs/350

DOI : $10.4000 /$ ccs.350

ISSN : 2558-782X

\section{Éditeur :}

Presses universitaires de Rennes, Association des lecteurs de Claude Simon

\section{Édition imprimée}

Date de publication : 8 juillet 2016

Pagination : 147-159

ISBN : 978-2-7535-4876-3

ISSN : $1774-9425$

Référence électronique

Michel Sandras, "Multiplicités : la série énumérative dans L'Acacia », Cahiers Claude Simon [En ligne], 11 | 2016, mis en ligne le 15 septembre 2017, consulté le 01 mai 2019. URL : http:// journals.openedition.org/ccs/350; DOI : 10.4000/ccs.350 


\title{
MULTIPLICITÉS : LA SÉRIE ÉNUMÉRATIVE DANS L'ACACIA
}

\author{
Michel SANDRAS \\ Université Denis Diderot-Paris 7
}

Au-delà de leurs différences dans la composition et dans l'emploi du matériel verbal, les romans de Claude Simon présentent une particularité d'écriture qui leur est commune: la série énumérative, les effets de liste donnés par les successions de groupes nominaux, verbaux ou de propositions brèves. Elles se combinent avec des phénomènes de répétition à différents niveaux et l'emploi massif du pluriel, ainsi qu'avec l'insistance sur tout ce qui est de l'ordre du comptable. Pour réunir tous ces traits je proposerai la catégorie de la multiplicité. Le texte romanesque est multiplicité par excellence, qui en accueille toutes les formes (ce qui rend compte de son hétérogénéité), sur le plan syntagmatique (ordre et liaison des épisodes) comme sur le plan paradigmatique (parallélismes dans les situations ou les personnages), qu'on le conçoive en termes de réseaux ou de modes d'assemblage. Cette catégorie me paraît particulièrement pertinente pour l'ensemble des romans de Claude Simon et à plus d'un titre: fabrication matérielle de ses livres par l'utilisation d'archives de nature diverse (lettres, cartes postales, photos, documents militaires) et par montage de fragments numérotés; composition au sens rhétorique (multiplicité des fils narratifs, rimes de situation) et au sens thématique: évocation récurrente de lieux, d'objets, de personnes, de destins. Mon propos est de voir comment la catégorie du multiple peut rendre compte de certains aspects de L'Acacia. Dans le cadre de cet article, je m’en tiendrai essentiellement à l'existence et au sens des séries énumératives présentes à de nombreuses reprises et sous des formes diverses - accessoi- 
rement à l'emploi du pluriel. Sans en faire une étude exhaustive ni entreprendre une comparaison systématique avec ses autres romans, je voudrais ici, après avoir proposé une typologie, avancer quelques réflexions sur les relations entre ces formes et les séquences où elles apparaissent, pour les rapporter à deux motifs du livre, le chaos et la similitude qui, sans être spécifiques à L'Acacia, y reviennent avec insistance.

\section{ESSAI DE TYPOLOGIE}

Dans les premières pages de son livre La Série énumérative, Béatrice Damamme-Gilbert ${ }^{1}$ rappelle quelques définitions traditionnelles de l'énumération. Celle des dictionnaires met le plus souvent l'accent sur le dénombrement (les diverses parties d'un ensemble). Pierre Fontanier la classe dans la catégorie "figures de style par emphase ${ }^{2}$ ", la définit par le terme « conglobation» (réunion sous un seul point de vue d'un plus ou moins grand nombre de traits sur le même sujet, ce qui l'apparente à l'accumulation) tandis que le groupe de Liège, plus attentif à la dimension linguistique, y voit l'acte de "déplier le syntagme par multiplication des aspects ou des attributs de l'un de ses lexèmes ${ }^{3}$ ". Madeleine Frédéric ${ }^{4}$ introduit un critère sémantique en distinguant l'énumération homologique et l'énumération chaotique tout en soulignant qu'elles ont en commun "l'expression analytique d'un ensemble » et l'existence d'un " terme-pivot» qui commanderait la série des syntagmes. L'auteur de La Série énumérative voulant mettre l'accent sur la construction retient finalement dans sa définition l'existence d'une succession d'unités linguistiques appartenant à des catégories morphologiques ou syntaxiques fonctionnellement équivalentes placées côte à côte 5 .

Si l'on s'en tient au genre du roman, on est forcé de reconnaître que la présence de séries énumératives est fort ancienne et très répandue et qu’elles prennent des formes et des significations différentes, selon leur fonctionne-

1. Béatrice Damamme-Gilbert, La Série énumérative. Étude linguistique et stylistique sappuyant sur dix romans français publiés entre 1945 et 1975, Genève, Droz, 1989.

2. Pierre Fontanier, Les Figures du discours, Flammarion, 1968 [1830], p. 363.

3. Groupe $\mu$, Rhétorique générale, Larousse, 1970, p. 77.

4. Madeleine Frédéric, La Répétition, étude linguistique et rhétorique, Tübingen, Max Niemeyer Verlag, 1985; M. Frédéric, "Énumération(s)/liste et monde du texte", dans Michelle Lecolle, Raymond Michel, Sophie Milcent-Lawson (dir.), Liste et effet liste en littérature, Classiques Garnier, 2013, p. 359377. Ce dernier ouvrage offre d'intéressantes contributions à l'étude des séries énumératives dans le roman, le poème, la chanson.

5. B. Damamme-Gilbert, op. cit., p. 37. 
ment morphosyntaxique, le nombre d'unités, le type de démarcation entre les unités, les rapports sémantiques, les effets stylistiques, leur appartenance au simple inventaire ou à une description plus ou moins étoffée, enfin selon leur place et leur rôle dans la narration.

Dans L'Acacia, toutes les catégories grammaticales sont représentées: substantifs (précédés ou non de déterminants), groupes nominaux en " de ", adjectifs détachés, participes présents, groupes verbaux et propositions. C'est évidemment la fréquence des participes présents qui attire l'attention:

des centaines de pieds, des centaines d'hommes errant comme au hasard, renvoyés d'un quai à l'autre, s'entremêlant, s'entrecroisant, rebroussant chemin, hâtant le pas, parfois même courant, se heurtant dans le noir, reprenant leur route, dans une sorte de morne, muet et docile tumulte (p. $1108^{6}$ )

ils descendirent d'abord vers le sud, chevauchant de nuit dans des montagnes, cantonnant dans des hameaux où vivaient des paysans sauvages, repartant, suivant des gorges, franchissant des ponts invisibles [...], longeant des fleuves laiteux, vaguement phosphorescents dans le noir (p. 1169)

C'est un trait bien connu du style de Claude Simon, dont les effets ont souvent été analysés: on ne s’y attardera pas.

Pour ce qui concerne la démarcation des unités, la plupart du temps les termes qui composent la série sont simplement juxtaposés et séparés par des virgules ${ }^{7}$. Quand la série a une certaine longueur, il arrive à Simon d'utiliser des parenthèses pour isoler certains segments et parfois à deux niveaux (parenthèses à l'intérieur de parenthèses ${ }^{8}$ ). Le doublement, voire le triplement du double point apparaît dans des énumérations apposées dont les termes appartiennent à des catégories sémantiques différentes (partie du corps/ terme général, concret/abstrait) :

quelque chose de vivant, mobile: crins, muqueuses, lèvres, salive, langues, yeux, voix, souffles: la chair sans mensonge, crédible, docile dans ses mains, se mouvant, s'écartant, s'ouvrant: la solitude, la mort, le doute conjurés, vaincus (p. 1241)

On trouve parfois au milieu de la série les expressions nominales, les adverbes spatiaux ou temporels qui traditionnellement servent à structurer une

6. Nous renvoyons à la pagination du vol. II de l'éd. des CEuvres de Claude Simon, établie par Alastair B. Duncan, Bérénice Bonhomme et David Zemmour, Gallimard, "La Pléiade », 2013.

7. Sur la ponctuation chez Simon, voir les travaux de Gérard Roubichou (Lecture de L'Herbe, Lausanne, L’Âge d'homme, 1976), de Stéphane Bikialo, "Les virgules de Claude Simon ", dans La Ponctuation, J. Dürrenmatt, La Licorne, n 52, 2000, p. 217-229 (en ligne sur le site de La Licorne, http:// licorne.edel.univ-poitiers.fr/index.php), et les pages que lui consacre Isabelle Serça dans son ouvrage Esthétique de la ponctuation, Gallimard, 2012.

8. Par exemple p. 1137. Mais la mise entre parenthèses a aussi d'autres fonctions. 
description ou un récit (à une extrémité, ailleurs, parfois, puis), les marqueurs additifs (aussi) et ceux qui indiqueraient un ordre dans la saisie d'un ensemble. Quelques suites affichent la présence de mais ou de pas, par exemple quand il s'agit d'une énumération par négation et ordonnée par gradation:

puis ils comprirent que ce n'était pas seulement le régiment, pas la brigade, pas l'arme particulière (la cavalerie) à laquelle ils appartenaient, pas la division, pas le corps d'armée, pas même l'armée à laquelle appartenait le corps d'armée, mais les armées voisines aussi, et qu'ensuite ce seraient les armées de réserve (s'il y en avait), et après les réserves tous ceux que l'on pourrait ramasser dans les dépôts, les ateliers, la moindre caserne, dans les parties les plus reculées du pays (p. 102899)

Par la rectification ou l'autocorrection la prose de Simon se révèle très soucieuse de mieux dire. À cette fin deux éléments s'intercalent parfois dans la construction asyndétique de l'énumération. L'un est l'indice de reformulation « ou plutôt » :

puis soudain les cris, les rafales des mitrailleuses, la tête de la colonne refluant, d'autres mitrailleuses alors sur l'arrière, la queue de la colonne prenant le galop, les cavaliers se mêlant, se heurtant, la confusion, le tumulte, le désordre, les cris encore, les détonations, les ordres contraires, puis lui-même [...] assourdi par les explosions, les cris, les galopades, ou plutôt percevant (ouie, vue) comme des fragments qui se succèdent, se remplacent, se démasquent, s'entrechoquent, tournoyants: flancs de chevaux, bottes, sabots, croupes, chutes, fragments de cris, de bruits, l'air, l'espace, comme fragmentés, hachés eux-mêmes en minuscules parcelles, déchiquetés, par le crépitement des mitrailleuses (p. 1060-1061)

L'autre est le comparatif "comme $\mathrm{si}^{10}$ " - tous deux sont des stylèmes propres à l'auteur - auxquels on ajoutera des "peut-être » dans les cas où l'énumération est présentée comme hypothétique (p. ex p. 1083-1084).

Les suites énumératives d'une certaine longueur utilisent la répétition lexicale et sont structurées par des constructions anaphoriques juxtaposées:

Il pleuvait sur les pans de murs des maisons éventrées dont les papiers aux couleurs pastel se décollaient peu à peu, il pleuvait sur la surface unie, grise et lente de la rivière où les gouttes faisaient éclore de petits ronds argentés, il pleuvait sur le paysage grisâtre, le cercle des collines sous lesquelles achevaient de pourrir les corps déchiquetés de trois cent mille soldats, sur les champs grisâtres, les maisons grisâtres - ou plutôt ce qu'il en restait, c'està-dire comme si tout, collines, champs, bois, villages, avait été défoncé (p. 1017)

Ou encore la page qui énumère les sujets des photographies prises par la mère:

9. Voir aussi p. 1106.

10. Voir Joëlle Gleize, "Comme si c'était une fiction. Sur un dispositif analogique dans L'Acacia de Claude Simon ", Michigan Romance Studies, 13, 1993, p. 81-102. Mais " comme si » est surtout une figure spécifique à l'art du prosateur qui cherche à mieux dire. 
Elle photographia les attelages au repos [...]. Elle photographia aussi des ours des Pyrénées [...]. Elle photographia l'automobile arrêtée... (p. 1081)

La répétition lexicale, qui n'est nullement requise dans l'énumération, constitue un outil rhétorique supplémentaire pour structurer des segments plus longs et dans certains passages (comme ceux cités plus haut) contribue à leur donner une allure lyrique.

La question de la taille des séries doit être articulée d'une part à celle de l'efficacité descriptive, d'autre part à celle du rythme de la prose. Il faut distinguer les séries qui se contentent de nommer des éléments et celles où la caractérisation plus étoffée amorce une description continue - et parmi ces dernières celles qui peu ou prou circonscrivent l'objet décrit et celles qui correspondent à une opération de glissement ou de dérive. La pure nomination fait se succéder des substantifs, parfois sans déterminant (p. 1241, voir supra):

la moitié de la maison dont il avait hérité (c'est-à-dire la moitié d'environ mille mètres carrés de bâtiments (remise, écurie, caves, escalier, véranda, salons, salles à manger, chambres, corridors, cuisines, lingeries, offices, galetas) entourant une cour, un jardin et une terrasse) (p. 1137)

ou des noms propres, qui indiquent la provenance des cartes postales, comme dans la série des lieux et monuments de Singapour (p. 1086-1087).

La plupart du temps la série contient des éléments descriptifs au moins ponctuels qui augmentent la taille des unités et peuvent donner l'aspect d'un tableau. Plus curieuse est l'énumération descriptive qui prolifere en ouvrant une digression ou qui se dirige vers un détail imprévisible. La description de la colonne des cavaliers, après avoir énuméré les traits de ressemblance, se termine par la perception d'une différence: la diminution conjointe du bruit de la pluie et de l'obscurité (p. 1164-1165). La longue série caractérisant de manière touristique la ville de Berlin (p. 1117) - ses « musées remplis de temples grecs ", " ses avenues de tilleuls ", " ses arcs de triomphe surmontés d'animaux de fer ", etc. - dérive vers la pâtisserie qui permet à l'ami mexicain de faire une plaisanterie - « Des Berlinoises savourant des Bavaroises! - contrepoint aux «types à araignées ${ }^{11}$ " qui « foutent salement la trouille»-mention qui, rétroactivement, inquiète l'énumération touristique. La page décrivant le parcours du régiment du père vers la gare (p. 1040), après avoir nommé les lieux et avant d'en arriver au geste patriotique et convenu (jet de fleurs) s'arrête à 
l'objet sexuel, dont les vieux messieurs ne seront pas privés à la différence des jeunes hommes qui partent à la guerre.

L'analyse des séries doit être attentive au terme pivot, ou thème-titre, à la formule qui indique le plus souvent au début (mais parfois à la fin) la nature de l'ensemble dont les éléments sont déclinés. Un passage du récit de l'embuscade ne propose aucun titre (p. 1061, voir supra) : le lecteur comprend que le narrateur énonce ce qu'il voit et ce qu'il entend successivement. Si certaines énumérations nomment concrètement le terme-pivot (support photographique ou ancrage géographique des vues), dans certains cas elles sont annoncées par une métaphore (p. 1027, en l'occurrence double métaphore, au début et à la fin de la série) :

cette toute puissance occulte et sans visage dans laquelle ils [les cavaliers de l'échelon de réserve] englobaient pêle-mêle généraux, politiciens, éditorialistes et tout ce qui touchait de près ou de loin à ce pandémonium

ou désignées par un nom propre dont la phrase a auparavant déplié les métonymies :

un fouillis de fougères arborescentes, de feuillages géants, vernis, charnus, rainurés, pendant en grappes, s'éparpillant, festonnés, gaufrés, striés, déchiquetés, aériens, s'entremêlant, se bousculant, entourant d'un cadre exubérant une muraille de roche, une cascade, un bassin d'où émerge le buste laiteux et nu d'un homme à la barbe carrée, les mains aux hanches ("Madagascar ») (p. 1056)

ou par des indéfinis comme "tout ", "quelque chose de" ou "quelque chose comme " et, trait plus curieux, "cela donc » ou " ceci donc » suivis d'un double point. Souvent ces énumérations ne retiennent que des segments synecdochiques ou métonymiques. Par exemple pour évoquer les circonstances des rencontres entre le militaire et sa future épouse, l'auteur imagine la première rencontre lors d'un mariage, puis présume et résume d'autres occasions en donnant une liste:

cela donc: quelque chose où il y eut des fleurs sur la table, du champagne dans les coupes, des parfums, des robes de soie, des habits noirs, des uniformes, des rires, des voix confuses, un orchestre peut-être, peut-être une valse, et peut-être non pas un rendez-vous mais quelques rencontres fortuites arrangées à la promenade ou à l'occasion de quelque concours hippique par de complaisants amis (p. 1083-1084)

Cet exemple va à l'encontre de l'énumération conçue comme définition à partir d'un terme englobant. La formule « cela donc », fréquente chez Simon, tait le nom de l'ensemble dont les composants sont déclinés. Le refus d'une 
nomination univoque ${ }^{12}$ (voir la répétition d'expressions comme quelque chose comme, une sorte de, une espèce de) joint à " donc ${ }^{13} "$ - moins une conjonction qu'un adverbe d'énonciation qui clôt une série et recentre un propos - coupe court à un développement, court-circuite une amplification.

Dernière remarque d'ordre formel: les séries énumératives sont composées le plus souvent d'unités au pluriel: pluriel grammatical, noms collectifs (magma, amas, débris, morceaux, colonne, foule, troupe), quantificateurs (comme certains déterminants indéfinis : beaucoup de, plusieurs) et certains noms employés métaphoriquement (" une avalanche de cartes postales»). Les textes de Claude Simon paraissent faire un usage massif du pluriel qui nappe de nombreuses descriptions, qui s'étale parfois sur des pages entières et sur lequel nous reviendrons. On conclura que la stratégie de l'énumération est renforcée par d'autres procédures, sémantique et grammaticale - l'emploi du pluriel - et rhétorique - la répétition lexicale, l’anaphore.

\section{CONTEXTES ET EFFETS DE SENS}

Dans L'Acacia, l'énumération doit être mise en relation avec la documentation utilisée par l'auteur, la nature des séquences et certains motifs spécifiques du livre, en particulier celui du chaos et celui de la similitude.

Et d'abord avec les archives photographiques ayant servi à sa composition, qu'à de nombreuses reprises l'auteur passe en revue. Certaines photos sont énumérées dans une perspective chronologique pour témoigner de la métamorphose du voyageur (p. 1055-1058). Les cartes postales qu'il envoie à sa future épouse (p. 1085-1087) ponctuent la géographie des déplacements du militaire et la progression d'une intimité. Mais comme elles montrent différentes villes avec leurs lieux et leurs monuments ou bien des paysages et des groupes humains qui témoignent d'une grande diversité exotique, leur accumulation finit par donner l'impression de fourre-tout, comme celle qui provient toujours de l'ouverture des boîtes où les familles les ont entassées sans nécessairement les dater. Un autre type de "désordre " est offert par la façon de présenter pêle-mêle les cartes amicales ou galantes reçues par

12. Voir l'étude de S. Bikialo, "La reformulation créative dans Le Palace: détournement de la reformulation et déroute de la nomination ", Semen, 12, 2000, p. 51-64 (en ligne: http://semen. revues.org/1874).

13. Ou " ceci donc". D. Zemmour, dans son livre Une syntaxe du sensible. Claude Simon et l'écriture de la perception (PUPS, 2008), a relevé l'importance de cet emploi de « donc ". 
« la jeune sultane » qu'elle a toutes conservées, celle de la grotte de Lourdes comme celle "des polissonnes petites femmes en culotte" (p. 1078).

L'effet de fatras est évidemment plus saillant pour les témoignages des guerres. Ainsi les cartes postales qu'envoie la veuve partie avec les deux sœurs et l'enfant à la recherche du corps de son mari :

À peu de choses près, elles auraient pu être toutes pareilles, la même presque, c'est-à-dire également grisâtres elles aussi, mal tirées sur des rectangles de mauvais carton, monotones, comme les collines, les ruines ou les étendues informes qu'elles représentaient, les débris qui bordaient les berges d'une rivière au cours rapide, à la fois silencieux et bruissant, coulant entre deux sillages d'épaves [...], partant d'un confus désordre de planches, de roues brisées, de timons et de blocs de pierre, aboutissant sur l'autre berge au même apocalyptique et fastidieux enchevêtrement de débris de voitures, de charpentes, de tuiles et de choses démantibulées (p. 1019-1020)

On relève dans cette suite un élément hétérogène: " la rivière au cours rapide, à la fois silencieux et bruissant ", oxymore et adynaton (détail impossible à voir), point de beauté et de poésie au milieu des ravages du temps et de l'histoire. Ces lignes montrent clairement le rapport entre la série énumérative et le motif du chaos, qu'on retrouve à de nombreuses reprises dans les épisodes de combats (p. 1060-1061, voir supra) ou le bric-à-brac propre à la déroute, à la désorganisation engendrée par la guerre ${ }^{14}$ :

la lente succession des véhicules hétéroclites (charrettes à foin, carrioles, tombereaux) couleur de terre (c'était quelque chose que même dans la demi-obscurité on pouvait voir, comme on peut sentir une odeur dans les ténèbres; quelque chose qui était inhérent aux voitures, aux ballots entassés, aux vêtements: le brun terne des couvertures, de la boue accrochée aux roues, des croûtes écaillées sur les jarrets des vaches et des veaux - seule parfois une tache noire trahissait le rouge d'un édredon ou d'une courtepointe), avec leurs chargements encordés et débordants, les bestiaux attachés par une longe à l'arrière, les femmes assises parmi les paquets, semblables elles-mêmes à des paquets (p. 1161)

Les énumérations de Claude Simon jouent à la fois sur la succession et sur la simultanéité, cette dernière ayant toujours le dernier mot puisque les cartes postales et les photographies, même si elles sont les traces de lieux datés, sont étalées et exposées en même temps. La série énumérative n’est que rarement temporelle, elle ne reproduit pas " des épaisseurs de temps »: même quand elle est constitutive d'un récit, elle met souvent en valeur le " en même temps ». Ce qui se trouve confirmé par les nombreuses références au mouvement des

14. Le chaos des civils en fuite inviterait à confronter les pages de Claude Simon à celles de Louis Calaferte dans C'est la guerre (Gallimard, coll. «L'Arpenteur », 1993) sur le double plan de l'énonciation et de la disposition typographique des séries. 
trains circulant en Europe qui rend compte d'une réalité historique, mais qui, faisant entendre le bruit de la mort, paraît arrêter le temps:

dans l'un de ces trains qui tous ensemble, au même moment, grondaient sur des ponts, s'engouffraient dans des tunnels, franchissaient des fleuves, sifflaient lugubrement, haletaient à travers les plaines d'un continent couturé de cicatrices (p. 1127)

Entre l'énumération et le pluriel qui peut affecter ses éléments, il n'existe pas de lien nécessaire - une série peut égrener des noms au singulier - mais des affinités dont l'œuvre entière de Claude Simon offre de nombreux exemples: les descriptions de lieux (le plus souvent urbains) qui associent les objets, les gens, éventuellement les animaux (LeVent, Histoire) ; les groupes : les voyageurs des trains (L'Acacia), le peloton des cavaliers, les prisonniers (Les Géorgiques, L'Acacia), les femmes (La Chevelure de Bérénice), les danseuses (Le Jardin des Plantes), les plantes et les oiseaux (Le Jardin des Plantes, Les Géorgiques), les inventaires: mobilier (Le Palace), comptes, mots (Histoire), photographies (Histoire, L'Acacia); et dans la plupart de ses romans, débris, détritus, épaves, fragments d'objets, bazars hétéroclites. L'emploi généralisé du pluriel est très perceptible dans les pages de L'Acacia évoquant la guerre, comme il l'était déjà dans Le Palace et Les Géorgiques. Rappelons que traditionnellement le pluriel littéraire est lié à la démesure, dont rendent compte les épopées ${ }^{15}$ antiques qui dénombrent volontiers les armes, les bateaux, les noms des chefs, les noms des morts. Mais il existe un autre emploi du pluriel qu'on peut nommer "critique ». Chateaubriand, dans un court texte intitulé "Orateurs ${ }^{16}$ " évoque une réunion du club des Cordeliers en 1792:

Les orateurs, unis pour détruire, ne s'entendaient ni sur les chefs à choisir, ni sur les moyens à employer; ils se traitaient de gueux, de gitons, de filous, de voleurs, de massacreurs, à la cacophonie des sifflets et des hurlements de leurs différents groupes de diables $[\ldots]$. Détruire et produire, mort et génération, on ne démêlait que cela à travers l'argot sauvage dont les oreilles étaient assourdies [...]. Des charpentes abattues, des bancs boiteux, des stalles démantibulées, des tronçons de saints roulés et poussés contre les murs, servaient de gradins aux spectateurs crottés, poudreux, soûls, suants, en carmagnole percée, la pique sur l'épaule ou les bras nus croisés.

Le pluriel généralisé a ici une valeur polémique: il souligne la ressemblance, l'indistinction des participants, ainsi que l'atmosphère de désordre et de destruction. Il semble que dans L'Acacia plusieurs séries énumératives couplées à

15. Et leur version parodique quand Voltaire, dans Candide, au début du chapitre 3, évoque la grande bataille entre le roi des Bulgares et celui des Abares.

16. Chateaubriand, Mémoires d'Outre-tombe, $1^{\text {re }}$ partie, livre IX, chap. 3, Le Livre de Poche, 1989, t. 1, p. $559-560$. 
un emploi généralisé du pluriel se trouvent accordées à cette valeur critique, notamment lorsque se rencontrent le motif du chaos et celui de l'indistinction.

L'autre motif qui engendre des énumérations renforcées par la répétition de certains mots est en effet celui du semblable, obsédant celui qui, dans Le Palace, donnait à l'homme-fusil " cette faculté de ceux pour qui le monde est partout et toujours le même [...] et qui est comme le contraire de la faculté d'étonnement ${ }^{17}$ ». Soit dans sa version simultanée - vêtements (vestons, casquettes et chemises des jeunes recrues regroupées au centre mobilisateur, juifs polonais vêtus de noir, calottes brodées des Soviétiques, hommes sanglés dans des uniformes bruns ou noirs à Berlin), gestes et équipement des cavaliers (p. 1164) - soit dans sa version successive - sœurs travaillant comme des mules, rituels des divertissements de la future épouse et de la vie du couple à Madagascar (p. 1098). Si la vision d'un monde fragmenté est commune à tous les livres de Claude Simon, l'originalité de L'Acacia paraît être la série qui, ponctuée par la reprise de l'adjectif "même ", souligne avec insistance la similitude, à la fois repoussante et attirante. Le retour du même et « la multitude de répliques d'un même type » (p. 1116) mettent l'accent sur l'indistinction, l'indiscernable. Valeur équivoque pouvant, selon les contextes, toucher au comique bouffon, au dérisoire, à l'amertume et à la fatigue mélancoliques, parfois à l'horreur ${ }^{18}$ : dans L'Acacia le semblable est lié à l'ironie tragique de l'Histoire et de l'histoire de la famille, mais aussi à une certaine perception du groupe ${ }^{19}$ quel qu'il soit, mélange de répulsion et de fascination, présent déjà dans de nombreuses pages du Palace. D’où la force et la beauté de l'événement singulier, de celui qui fait la différence: le " coït aérien » (véritable hapax dans l'œuvre de Simon), le chant du coucou, la petite fille au chien, l'évasion en plein jour dans « le tranquille chuintement du vent dans les cimes

17. Claude Simon, Euvres I, éd. Alastair B. Duncan, avec Jean H. Duffy, Gallimard, "La Pléiade", 2006, p. 443-444.

18. Comme la carte postale envoyée par l'épouse à sa mère représentant trois Noirs: " "on se demande si ce sont des créatures humaines comme nous" "Le narrateur ajoute: "trois squelettes plutôt (ou échalas, ou épouvantails), trois choses hybrides, à mi-chemin entre le végétal et l'humain, c'està-dire où l'on ne distinguait pas très bien ce qui appartenait à l'un ou l'autre règne " (p. 10891090). Voir aussi les jeunes recrues dans le train, «semblables [...] à des créatures à mi-chemin entre l'homme $[\ldots]$ et ces bêtes à carapace à l'intérieur violacé composé d'un élémentaire système digestif et d'un élémentaire relais de neurones " (p. 1132). Comme l'écrit Michel Deguy, " [l]'œil qui regarde est un œil agrandi d'horreur " ("Claude Simon et la représentation (Sur Le Palace) ", Critique, 187, décembre 1962, p. 1024; en ligne sur le site de l'Association des Lecteurs de Claude Simon, http:// associationclaudesimon.org/).

19. À remarquer que pour Claude Simon il y a groupe dès qu'il y a trois unités. La figure ternaire, familiale et féminine, revient aussi dans les épisodes de la guerre. 
des pins » (p. 1228) - même quand il signe la folie comme la mort du colonel, "le sabre levé dans le soleil » (p. 1200).

\section{POUR CONCLURE}

Tout d'abord, la série énumérative, quelle qu'elle soit, repose sur ces deux principes: la fragmentation qui fait se succéder les unités du matériel verbal qu'elle segmente, et la ressemblance produite par l'équivalence fonctionnelle de ces unités, parfois doublée de répétitions lexicales ou rhétoriques. Or il faut souligner que segmentation et ressemblance sont susceptibles de prendre deux valeurs opposées. L'extrême morcellement du texte, qui donne aux énumérations des propriétés visuelles qui, sans être aussi saillantes que sous la forme de la liste verticale, ont néanmoins un pouvoir diagrammatique ${ }^{20}$, renvoie autant à une esthétique du discontinu propre à l'art moderne (poétique, pictural, musical) qu'à une perception chaotique et inhospitalière du monde. Et la ressemblance n'a pas seulement une valeur négative: elle est aussi constitutive de la lexis poétique depuis Aristote, qui la dit propre à percevoir le semblable dans les choses éloignées ${ }^{21}$ (par la métaphore et, ajouteront Valéry et Jakobson, la paronomase, la confusion entre le son et le sens). Principalement et localement, elle correspond au phénomène des "rimes ", entendu à la manière de Raymond Queneau, comme un dispositif de construction romanesque, essentiel dans les romans de Claude Simon.

D'autre part, on peut distinguer deux stratégies d'écriture dans L'Acacia. Le philosophe et poéticien Pierre Alféri oppose la consistance à la coupe ${ }^{22}$ en affirmant que la première est le souci majeur du prosateur et le choix judicieux de la seconde celui du poète (en vers). L'obtention de la consistance est nécessairement visée par le travail de la prose romanesque, sur le plan de la composition générale (montage des épisodes), de l'entrelacement des motifs, mais aussi de l'organisation des phrases, de leur enchainement, de leur rassemblement en paragraphes, voire de la disposition typographique. Tous les grands romanciers, Flaubert et Proust en particulier, ont accordé à ces questions la plus grande importance, proposant les métaphores les plus

20. Sur la dimension iconique ou diagrammatique de certaines formes linguistiques, voir l'article de Roman Jakobson "À la recherche de l'essence du langage ", dans Problèmes du langage, ouvrage collectif, Gallimard, coll. «Diogène », 1966, p. 22-38.

21. Conception contestée par l'art qui fait une place à l'incongruité et à la dissemblance (certaines formes de comique, le surréalisme).

22. Voir Pierre Alféri, «Vers la prose », remue.net:http://remue.net/spip.php?article3751. 
diverses et parfois les plus contradictoires (le mur, le ciment et le collier de perles pour le premier dans sa correspondance, le bœuf mode, la robe ou la cathédrale pour le second, à la fin du Temps retrouvé). La consistance de L'Acacia est produite au niveau paradigmatique par les rimes de situations - par exemple les scènes ferroviaires, les scènes de foules, les épisodes équestres -, par une construction soucieuse de ménager non une progression mais des échos; et au niveau syntagmatique par un emploi massif du pluriel, qui prend une valeur quantitative mais aussi qualitative. De plus l'auteur de L'Acacia se montre préoccupé par la consistance pour des raisons éthiques: réduire l'écart entre l'expérience vécue et le texte qui prétend en rendre compte (p. 1187). Mais, pour réduire cet écart, et là encore avec un souci d'authenticité, il y a une autre stratégie d'écriture, qui rappelle celle de la coupe évoquée par Alféri: la répétition des énoncés fractionnés, traduisant les bribes de souvenirs comme la diversité et la dissociation des émotions, visant moins à faire que la prose " prenne »- comme on dit de la confection du mortier ou d'une mayonnaise - qu'à faire entendre non sa musique mais son bruit et ses à-coups, dont la répercussion de wagon à wagon de l'entrechoquement des tampons et des attelages pourrait bien être l'emblème ${ }^{23}$.

Ces deux stratégies d'écriture sont utilisées en même temps, pour la raison que donne l'auteur - dire le "comment était-ce? ", pour reprendre la formule du Palace -, mais aussi donner la figure d'un destin que pourrait exprimer la célèbre formule Mané, Thécel, Pharès. En ce sens, l'énumération relèverait aussi de la sanction (la division) et du bilan, au sens comptable du $\operatorname{mot}^{24}$ : ce qui expliquerait le nombre d'informations données dans ce roman (pertes à la guerre, kilomètres parcourus, dépenses des paysans et surtout décompte des années, parfois moins exact que symbolique, tant pour la vie du père que pour celle du fils).

Dire "comment était-ce?": un certain nombre de textes autobiographiques du $\mathrm{Xx}^{\mathrm{e}}$ siècle ont affronté cette épreuve, d'autant plus redoutable quand le mince pronom neutre " $\mathrm{ce}^{25}$ " correspond à une expérience traumatisante. Pour ne pas trop nous éloigner de celle de Simon, citons Dialogue avec la mort d'Arthur Koestler incarcéré par les franquistes avec un régime

23. Phénomène sonore souligné plusieurs fois dans le livre.

24. L'Acacia est aussi le bilan d'une vie: qu'ai-je fait de tout ce temps? Comment continuer à vivre? Autant de questions posées parT. S. Eliot dans les Quatre quatuors, dont une citation sert d'épigraphe au roman.

25. Beckett aurait plutôt écrit « Comment c'était ». En plaçant le démonstratif en position finale, Simon retire à la consonne son appui vocalique postérieur. 
de condamné à mort, et Cellule 89 de Victor Klemperer, arrêté par les nazis, tous deux tenant un journal enregistrant des impressions et les mouvements de leur pensée ${ }^{26}$. Ou, dans une perspective cette fois romanesque, Pierre Guyotat, dont l'expérience de la guerre d'Algérie a inspiré Tombeau pour 500000 soldats. Ces ouvrages n'affrontent pas seulement la difficulté - traditionnelle - à dire le réel, mais aussi le rapport problématique du sujet à ce qu'il a vécu. À cette fin leurs auteurs respectifs ont creusé ou bouleversé la langue à la manière des poètes, ou imaginé des dispositifs singuliers d'énonciation et parfois de typographie. Quelques lecteurs de Claude Simon s'étonneront peut-être que, sur ce point, opérant avec la distance du pronom « il » et les séries énumératives (qui mettent en question la direction de la phrase et la prévision de sa clôture, mais pas sa structure), L'Acacia paraisse en retrait de certaines pages des Géorgiques et du Jardin des Plantes. Ils seront conduits à estimer que ce roman révélant beaucoup de maîtrise se tient sous la protection de la langue: signes qui sont toujours ceux de l'œuvre qu'on nomme « classique».

26. Voir sur ces deux textes l'étude de Carine Trévisan, «Arthur Koestler, Dialogue avec la mort: "L’ange distributeur des pensées" ", Textuel, n 67, "Littérature au présent. Mélanges offerts à Francis Marmande », Sylvie Patron (dir.), 2012, p. 277-288. 